\title{
Reducing contacts to stop SARS-CoV-2 transmission during the second pandemic wave in Brussels, Belgium, August to November 2020
}

Brecht Ingelbeen ${ }^{1}$, Laurène Peckeu ${ }^{1}$, Marie Laga ${ }^{1}$, Ilona Hendrix² , Inge Neven² , Marianne AB van der Sande ${ }^{1,3}$, Esther van Kleef ${ }^{1}$

1. Department of Public Health, Institute of Tropical Medicine, Antwerp, Belgium

2. Department of Infectious Disease Prevention and Control, Common Community Commission, Brussels-Capital Region, Brussels, Belgium

3. Julius Center for Health Sciences and Primary Care, Utrecht University, Utrecht, the Netherlands

Correspondence: Brecht Ingelbeen (bingelbeen@itg.be)

Citation style for this article:

Ingelbeen Brecht, Peckeu Laurène, Laga Marie, Hendrix Ilona, Neven Inge, van der Sande Marianne AB, van Kleef Esther. Reducing contacts to stop SARS-

CoV-2 transmission during the second pandemic wave in Brussels, Belgium, August to November 2020. Euro Surveill. 2021;26(7): pii=2100065. https://doi.

org/10.2807/1560-7917.ES.2021.26.7.2100065

To evaluate the effect of physical distancing and school reopening in Brussels between August and November 2020, we monitored changes in the number of reported contacts per SARS-CoV-2 case and associated SARS-CoV-2 transmission. The second COVID-19 pandemic wave in Brussels was the result of increased social contact across all ages following school reopening. Physical distancing measures including closure of bars and restaurants, and limiting close contacts, while primary and secondary schools remained open, reduced social mixing and controlled SARS-CoV-2 transmission.

Belgium reported per capita the highest number of coronavirus disease (COVID-19)-related deaths and near highest number of cases worldwide and was heavily affected during Europe's first and second pandemic wave, reporting a total of 21,634 deaths and more than 700,000 cases on 13 February 2021 [1]. We describe the effect of physical distancing and school reopening on the number of contacts reported by each confirmed case of severe acute respiratory syndrome coronavirus 2 (SARS-CoV-2) and on associated age-specific SARS-CoV-2 transmission patterns, using operational data from the COVID-19 contact tracing system of the Brussels region (Supplementary material) and case reports made available via the Belgian institute for health, Sciensano.

\section{Physical distancing measures in summer and autumn}

An increase in COVID-19 cases in July 2020 in Antwerp, Belgium's second largest city, was reverted following a provincial ban on indoor events involvingmore than 100 people, a curfew, mandatory teleworking, mandatory wearing of face masks, and a national limit of five close contacts per household. Close contacts were individuals outside the household, with whom one could have contact for more than 15 min without keeping a distance of $1.5 \mathrm{~m}$ and not wearing a mask, excluding children younger than 12 years. However, soon after the end of the summer holidays, while case numbers were rising again, national and regional governments loosened physical distancing measures. Belgium's Brussels-Capital region was first to observe a steep increase in cases but also to re-introduce physical distancing measures (Table).

\section{The second COVID-19 pandemic wave in Brussels region}

From 1 August to 12 November 2020, Brussels-Capital region reported 63,838 confirmed SARS-CoV-2 cases (5.2\% of a population of over 1.2 million [2]), i.e. RT-PCR positive, among 415,412 SARS-CoV-2 PCR tests performed. The daily number of confirmed cases peaked on 20 October with 2,950 reported cases (Figure 1). SARSCoV-2 test positivity was highest among 20-29-yearolds $(7.4 \%, 13,436 / 181,940)$ and decreased with age to $4.3 \%$ positivity $(4,913 / 114,637)$ among those 70 years and older (Supplementary Figure S1).

\section{Effect of physical distancing measures on the number of reported contacts of cases} We compared differences in the mean weekly number of contacts reported per case to the telephone- and field agent-based contact tracing system, and confidence intervals $(\mathrm{Cl})$, assuming normality, at the start and end of each intervention period (Table). Following school reopening on 1 September, the mean number of reported contacts per case increased from 2.01 (95\% $\mathrm{Cl}: 1.73-2.29)$ in the last week of August to 2.83 (95\% $\mathrm{Cl}: 2.59-3.06)$ in the first week of September and further increased to $3.04(95 \% \mathrm{Cl}: 2.93-3.15)$ by 30 September when the limit on the number of close contacts was suspended (Figure 2). A restriction to three close contacts and closure of bars on 6 and 8 October 
Physical distancing measures and SARS-CoV-2 testing policy changes, Brussels region, Belgium, July-November 2020

\begin{tabular}{|c|c|}
\hline Intervention & Start date \\
\hline Cafés and restaurants may remain open until 1:00 and can take maximum 10 people per group & 8 June \\
\hline Sports allowed in groups of maximum 50 people & 8 June \\
\hline Maximum five close contacts ${ }^{\text {a }}$ per week & 30 July \\
\hline Reopening primary and secondary schools & 1 Sep \\
\hline Restart universities at $50-75 \%$ room occupancy, with face masks & 14 and 21 Sep \\
\hline Limit on number of close contacts suspended & 30 Sep \\
\hline Quarantine for high-risk contacts ${ }^{\mathrm{b}}$ reduced from minimum 10 days to 7 days (if two negative tests) & 30 Sep \\
\hline Maximum three close contacts per week & $60 \mathrm{ct}$ \\
\hline Recommended teleworking & $60 \mathrm{ct}$ \\
\hline Bars and cafés closed at 23:00 & $60 \mathrm{ct}$ \\
\hline Bars and cafés closed & $80 \mathrm{Oct}$ \\
\hline Universities restrict seat occupancy to $20 \%$ & 19 Oct \\
\hline Testing restricted to symptomatic suspected SARS-CoV-2 cases (except for healthcare workers) & 21 Oct \\
\hline Quarantine for high-risk contacts extended to 10 days & 21 Oct \\
\hline Restaurants closed & $260 \mathrm{ct}$ \\
\hline $\begin{array}{l}\text { Maximum one close contact outside the household per person and maximum four people in private gatherings } \\
\text { (excluding } 12 \text {-year-olds) }\end{array}$ & $260 \mathrm{ct}$ \\
\hline Curfew between $10: 00$ and 18:00 & 26 Oct \\
\hline Teleworking becomes the rule & $260 \mathrm{ct}$ \\
\hline Indoor sports prohibited (except<12-year-olds) & 26 Oct \\
\hline Universities gradually switch to online learning & 26 Oct \\
\hline Maximum one close contact outside the household per household & $2 \mathrm{Nov}$ \\
\hline Mandatory teleworking & $2 \mathrm{Nov}$ \\
\hline Non-essential shops closed; professions involving physical contact or gatherings suspended & $2 \mathrm{Nov}$ \\
\hline Extended autumn school holiday ( 31 Oct-15 Nov) & 31 Oct \\
\hline
\end{tabular}

a Close contacts are persons who are not part of your household, with whom contact can last for more than 15 min without keeping a distance of $1.5 \mathrm{~m}$ and not wearing a mask, excluding children younger than 12 years.

${ }^{b}$ High-risk contacts are persons with whom the SARS-CoV-2 PCR-positive case had physical or cumulative 15 min non-physical contact within $1.5 \mathrm{~m}$ from 2 days before to 7 days after onset of symptoms.

Sources: https://www.commissioner.brussels/updates-covid-19; https://covid-19.sciensano.be/nl

resulted in a gradual decrease in reported contacts per case from a mean of 2.81 (95\% Cl: 2.74-2.89) in the first week to 2.21 (95\% Cl: $2.16-2.25) 2$ weeks later, just before contacts were further limited on 26 October. Following a limit to one close contact and closure of restaurants and sports facilities, the number of contacts per case further decreased to 1.94 (95\% Cl: $1.90-$ 1.99), a $36.2 \%$ decrease compared with 30 September. When also shops were closed, teleworking became mandatory and schools started the autumn break, the mean number of reported contacts stagnated at 1.85 (95\% Cl: 1.78-1.91).

The number of reported contacts per case was highest among 10-19-year-olds during our study period (3.11; 95\% Cl: 3.01-3.21); adults 70years and older reported the lowest number $(2.05 ; 95 \% \mathrm{Cl}: 1.93-2.18)$. However, over time, changes in the number of contacts following changes in physical distancing measures were largely similar across age groups, with the exception of the 0-9-year-olds (no changes observed) and adults 70 and older (less pronounced, Figure 2). Of note, testing and related contact tracing for 0-6-year-olds was restricted to symptomatic cases only during the period of study.

\section{Effect of the number of reported contacts per case on SARS-CoV-2 transmission}

We derived the instantaneous reproduction number $R_{t}$ i.e. the mean number of secondary cases that would arise from a primary case on a given day, from the daily number of reported cases, assuming an uncertain serial interval distribution (i.e. drawn from multiple truncated normal distributions with mean 5.19 days, 95\% credible interval $(\mathrm{Cr}):$ 4.37-6.02), setting a 7-day sliding window, and estimating $\mathrm{Crl}$ using bootstrapping [3]. The $R_{t}$ peaked on 17 September at 1.48 (95\% Crl: $1.35-1.63)$. Three weeks after the gradual restriction of close contacts (first three, then one) and the closure of bars, restaurants and sport facilities, $R_{t}$ had decreased by $44.6 \%$ to 0.82 (95\% Crl: 0.79-0.85) (Figure 3). Even though a change in testing strategy to symptomatic cases only might have contributed to the drop in $R_{t}$, the drop continued in the 2 weeks following the change. 
7-day moving average of SARS-CoV-2 confirmed cases reported, Brussels region, Belgium, 1 August-12 November 2020 $(\mathrm{n}=415,412)$

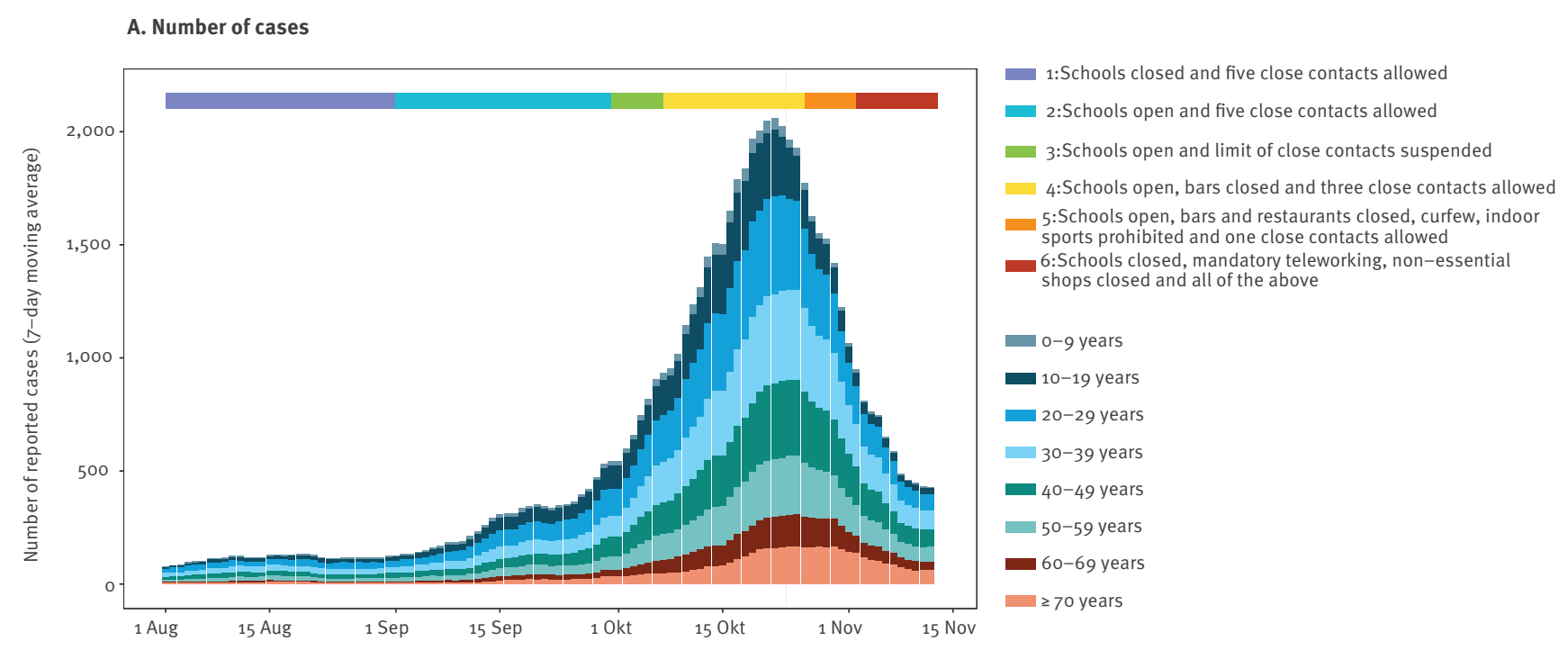

B. Percentage of reported cases per age group over time

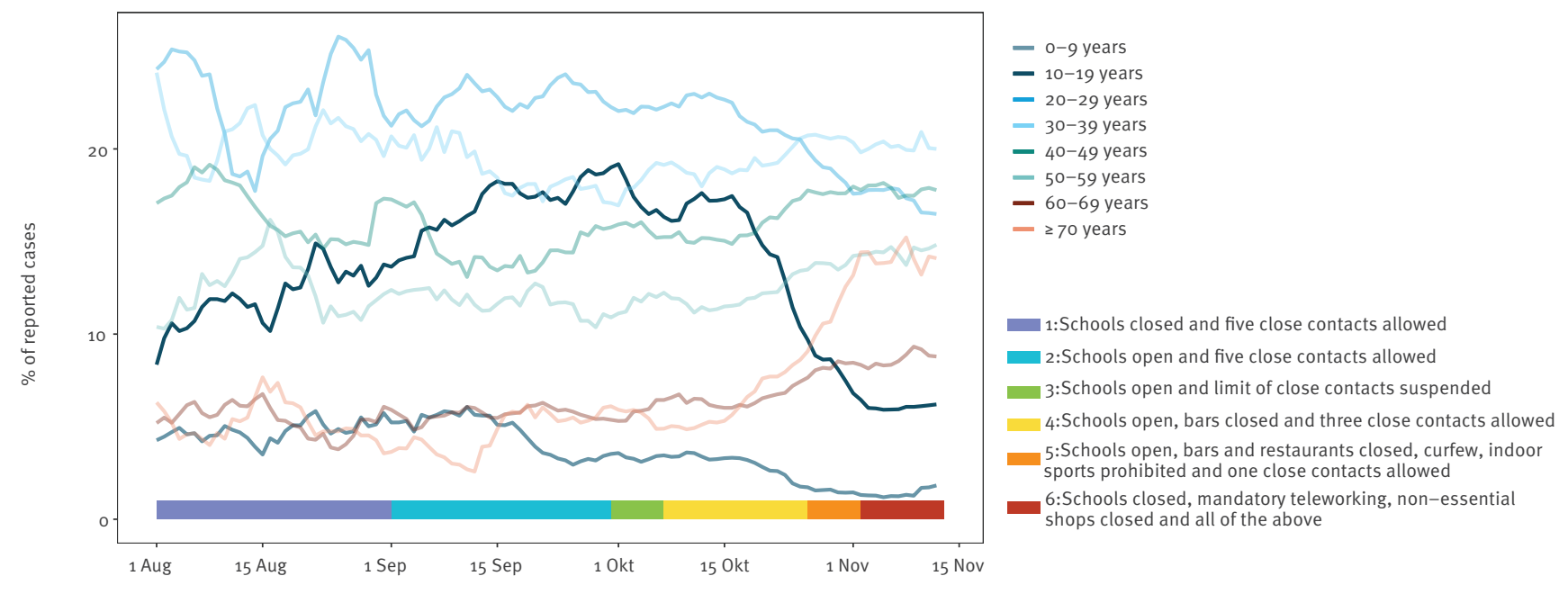

\section{Age-specific transmission patterns}

Among 2,387 primary-secondary case pairs identified during the period 1 August to 30 November, transmission within the same age group was predominant $(33.4 \%, 797 / 2,387)$. Infections originating from 10-19-year-olds were seldom recorded in August and November when schools were closed but testing of this group was low at these times as well (Figure 4A, Supplementary Figure S3). After schools reopened, transmission between all age groups became more apparent. In the month after reopening schools, $8.9 \%$ (67/755) of infections were from 10-19-year-olds to other age groups and $17.4 \%(131 / 755)$ from other age groups to 10-19-year-olds (Figure 4B). During extended autumn holidays and the closure of all non-essential services starting on 2 November (Figure 4D), intragenerational transmission was highest at 39.4\% (63/160). Transmission within older age groups ( $\geq 50$ years) became more frequent later in the second pandemic wave.

\section{Age-specific trends in reported SARS- CoV-2 cases}

SARS-CoV-2 case reports among 10-19-year-olds increased throughout August and September (Figure $1 \mathrm{~B})$, coinciding with an increasing testing rate in this age group (spearman rank correlation coefficient $=0.74 ; p$ value 0.001 ; Supplementary Figure S2). At the time schools reopened (1 September), we did not observe any significant change in the proportion of 10-19-year-olds among all diagnosed cases (adjusted for 4 days reporting delay; Poisson regression risk ratio 1.23; $95 \% \mathrm{Cl}$ : 0.79-1.94; Supplementary Figure S5). When asymptomatic contacts were excluded from SARS-CoV-2 testing (from 21 October onwards), the proportion of $10-19$-year-olds fell from $16.9 \%$ of cases $(3,478 / 20,535$ during the 2 weeks preceding the change) to $9.9 \%(2,214 / 22,330$ during the 2 weeks following the change, Figure $1 \mathrm{~B}$ ). The proportion of adults 70 years or older who tested positive increased from $5.2 \%(727 / 13,872)$ during the first 2 weeks of 


\section{FIGURE 2}

Weekly mean number of contacts reported per SARS-CoV-2 case (excluding cases not reporting any contacts), by age group, Brussels region, Belgium, 1 August-12 November $2020(\mathrm{n}=24,166)$

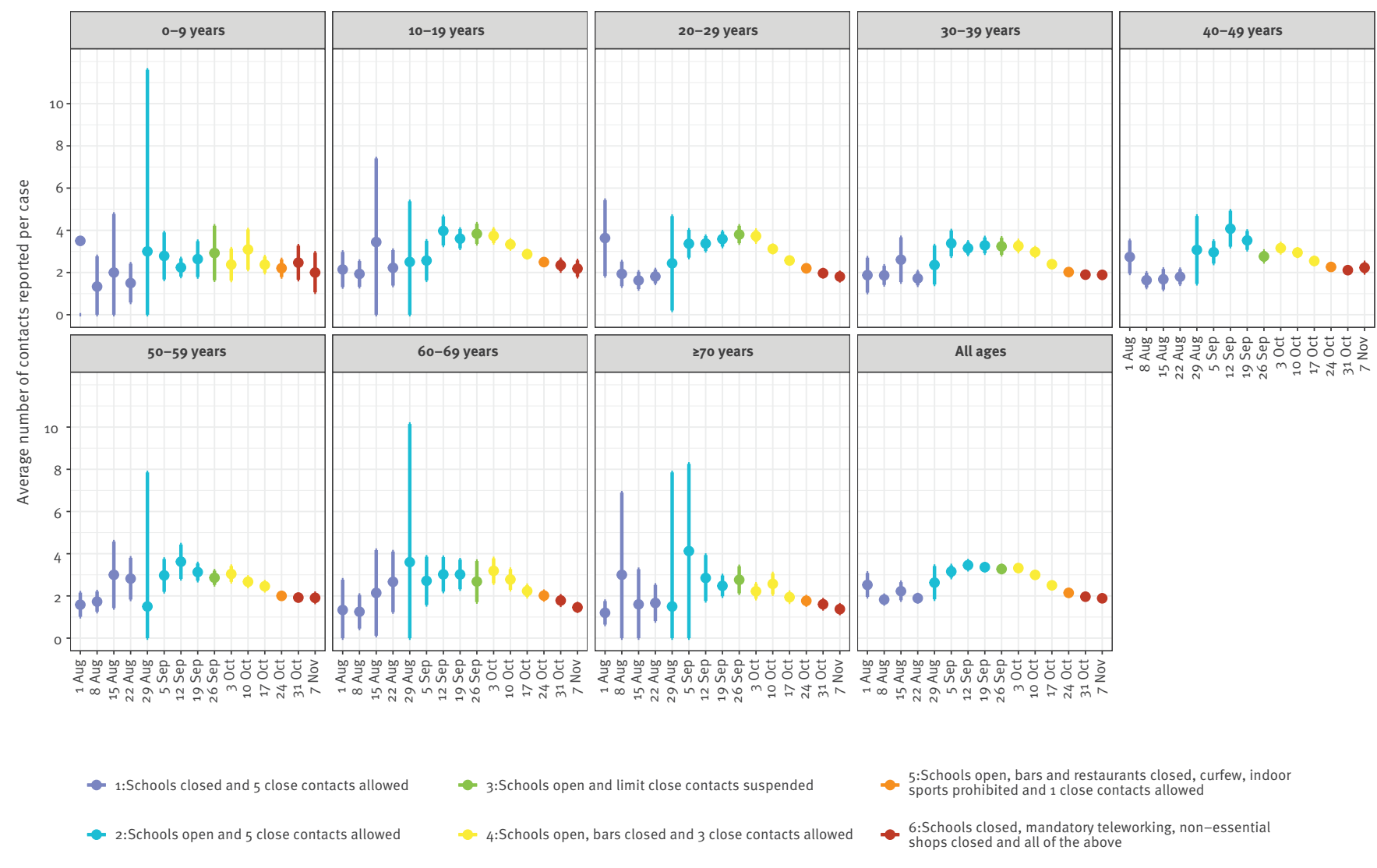

Weeks follow 7-day intervals from 1 August. Vertical bars indicate $95 \%$ confidence intervals. Colours indicate ongoing interventions or the intervention started that week. For trends in daily estimates and exact timing, see Supplementary Figure S2. For readability, the wide confidence intervals of the observation of the first week in the age group $0-9$ years were removed. Of note, testing and related contact tracing for 0-6-year-olds was restricted to symptomatic cases only during the period of study.

\section{FIGURE 3}

Estimated instantaneous reproduction number $R_{\mathrm{t}}$ based on daily reported cases and a mean 5.2-day serial interval (95\% credible interval: 4.4-6.0 [14]), Brussels region, Belgium, 1 August-12 November $2020(\mathrm{n}=63,838)$

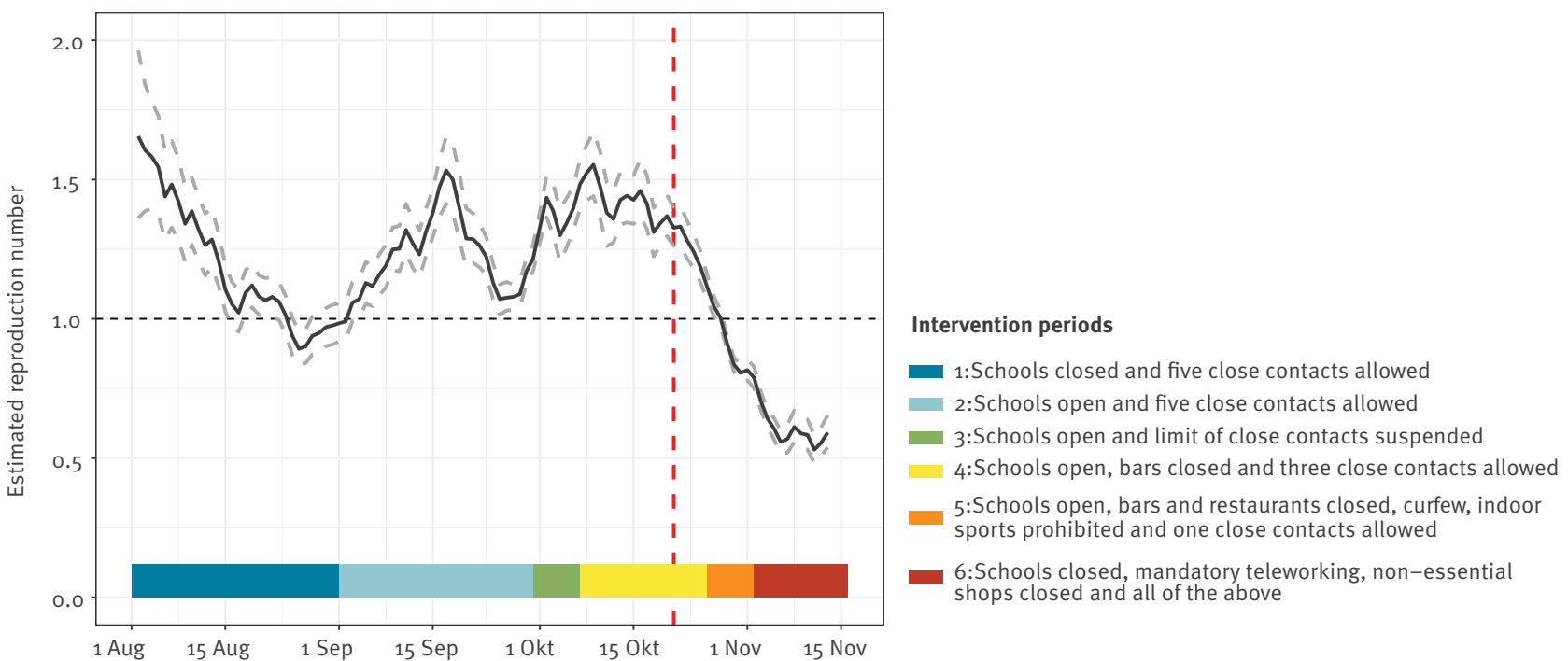

After 21 October (vertical dashed red line) asymptomatic contacts were excluded from SARS-CoV-2 testing. The dashed grey lines are $95 \%$ credible intervals. The horizontal dashed black line corresponds to a reproduction number of 1.

The analysis was done using the EpiEstim R package [3]. 
Transmission matrix between primary and secondary cases of all identified transmission events, Brussels region, Belgium, 1 August-30 November $2020(n=2,387)$
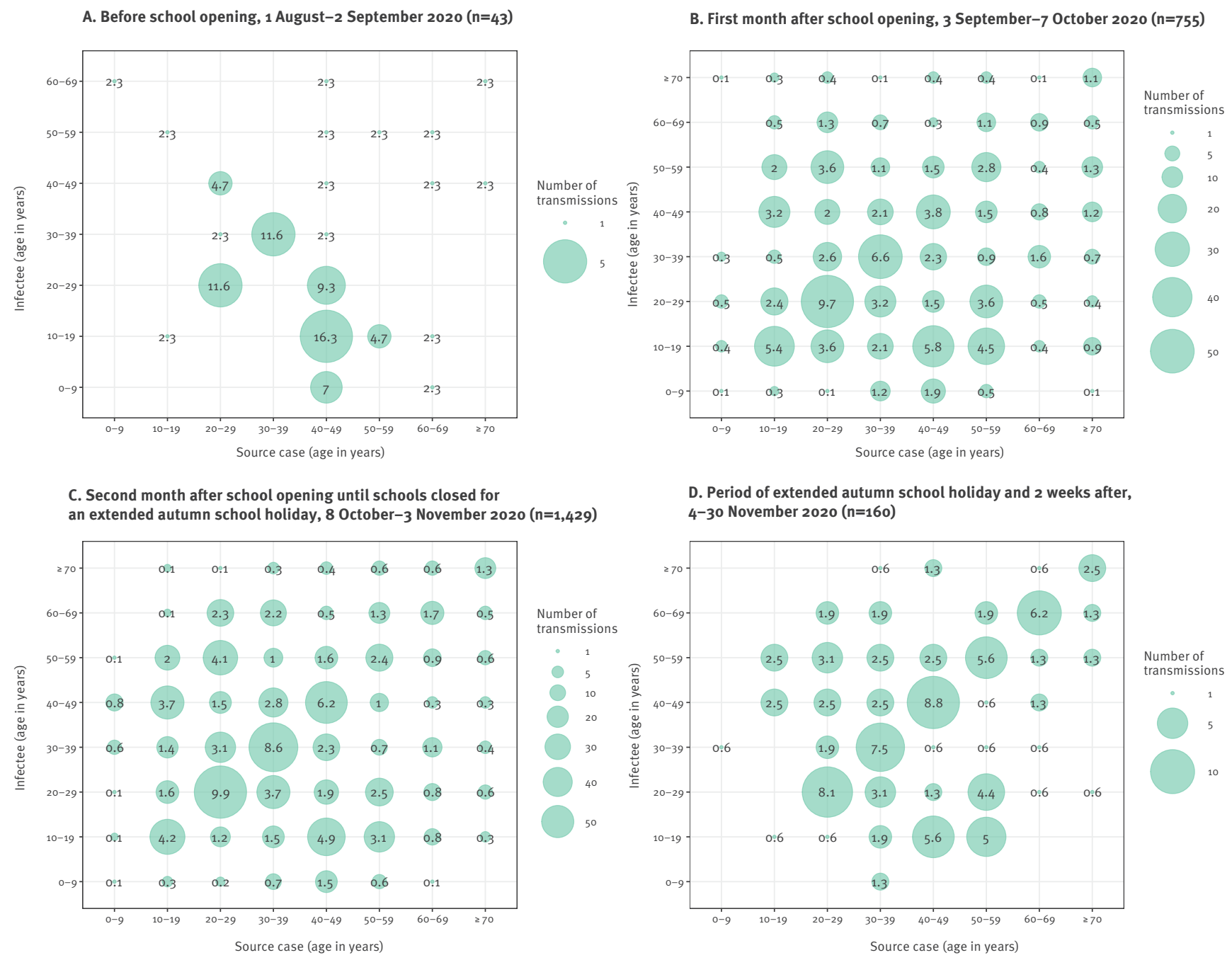

October to $13.8 \%(1,574 / 11,430)$ in the first 2 weeks of November (Figure 1B).

\section{Ethical statement}

The study was approved by the Institutional Review Board of the Institute of Tropical Medicine (reference number 1423/20) and the Ethics committee of the Antwerp University Hospital (reference number 20/34/435).

\section{Discussion}

September 2020 saw a persistent increase in SARS$\mathrm{CoV}-2$ cases in the Brussels region following increased social mixing across all ages, as inferred from trends in the number of reported contacts per case. Stringent physical distancing measures were introduced 1 month after a persistent increase in $R_{t}$. These initial measures (a limit to three close contacts per person, a curfew, closure of bars and recommended teleworking) reduced reported contacts of cases by more than a third within 3 weeks, resulting in an $R_{f}<1$ by 29 October; they reduced social mixing sufficiently to

control transmission, even with high case numbers and without closing schools or full lockdown.

In contrast to the first pandemic wave, primary and secondary schools remained open throughout the second wave. There is general consensus that children attending primary school contribute little to transmission [4]. In contrast, the role of teenagers and secondary schools is still much debated. Teenagers can transmit and show a viral load comparable to adults [5]. Nonetheless, several studies indicated either lower susceptibility or a higher proportion of asymptomatic individuals among teenagers which might result in fewer secondary infections originating from younger individuals [4,6-8]. Modelling studies investigating the role of secondary schools have shown that school closures can help alter transmission dynamics - albeit insufficiently for control and based on data from the first months of the pandemic with limited preventive measures in schools [e.g. 7,9,10]. Our findings confirm transmission among and from teenagers, with intergenerational transmission apparent following school 
opening. Nonetheless, their relative role was limited: transmission events from 10-19-year-olds to other age groups remained fewer than those from adults, and the proportion of cases among 10-19-year-olds did not significantly change after school reopening. After school reopening, the number of reported contacts per case increased across all age groups, suggesting a change in behaviour and mobility of all age groups, which may, at least in part, indirectly relate to school opening, and resulting in transmission particularly within the individual age groups, and an increased $R_{t}$.

Epidemic growth among older adults was delayed when compared to that in younger age groups, similar to observations in other European countries. Transmission of SARS-CoV-2 varies between age groups and settings [11]. In a socially structured disease system, transmission of infectious agents among individuals with social networks less linked to the general population (e.g. nursing home residents) can increase disproportionately when a network-specific abundance threshold, which may be different from the conventional $R>1$ for the spread of infections, is reached [12]. We hypothesise that this so-called percolation phenomenon may explain why transmission among older adults peaked later.

Our study had some limitations. Firstly, the number of reported contacts per case was smaller (mean in August: $2.0 ; 95 \% \mathrm{Cl}: 1.8-2.0$ ) and less heterogenous than what participants in a Belgian contact survey reported (mean: 3.5 during the period 27 July to 10 August) [13]. This was probably a result of our analyses only considering high-risk contacts (physical or cumulative $15 \mathrm{~min}$ non-physical contact within $1.5 \mathrm{~m}$ ) and a result of poor recall of context-specific accidental social contacts (e.g. public transport, bars) or reluctance to report contacts. Yet, age-specific differences were comparable, suggesting that the conclusions based on trends over time remain valid. Secondly, only a small proportion of cases were known contacts, indicating high volumes of undetected transmission or poor linking between data. Thirdly, a shift in testing policy to include only symptomatic cases from 21 October onwards is likely to have resulted in fewer identified transmission events involving children or teenagers because these groups more frequently present without or with mild symptoms. This shift in testing could have resulted in an underestimation of $R_{t}$ at the end of October. However, $R_{t}$ continued to decrease steadily after the change in testing strategy, suggesting that a true drop in transmission levels is likely. To determine a causal relationship between measures implemented and social mixing, in turn affecting SARS-CoV-2 transmission, we made sure we observed a strong correlation, coherence between the different analyses in the study, that no other change in policy or context could explain the effect observed, that the observed effect followed the introduction of a measure, and that there was a dose-response relationship such as between the number of contacts and Rt. Moreover, our findings are plausible, and are consistent with prior modelling and real-world studies on COVID-19 and other infectious agents.

\section{Conclusion}

The second pandemic wave in Brussels was a result of increased social mixing across all ages in the absence of strict physical distancing measures. Limiting the number of close contacts per person and closure of bars and restaurants resulted in a rapid decrease in reported contacts of cases, sufficient to control SARSCoV-2 transmission (lowering $R_{t}$ to $<1$ ).

\section{Acknowledgements}

We thank the Common Community Commission for reaching out to collaborate and providing insight at several stages of the study, and David Hercot for helpful last-minute discussions. We thank the contact tracing team for data collection, and Sciensano for making age-specific case reports and testing data open access.

Funding: This study was partially funded by EU grant 874850 MOOD and is catalogued as MOOD [004]. The contents of this publication are the sole responsibility of the authors and do not necessarily reflect the views of the European Commission.

Conflict of interest

None declared.

Authors' contributions

$\mathrm{BI}$ and EvK conceptualised the design and analyses and wrote the draft manuscript; LP contributed to the data analysis and interpretation; IN supported the interpretation of results and revised the manuscript; $\mathrm{IH}$ did the data curation and revised the manuscript; ML revised the manuscript; MvdS contributed to the interpretation and revised the manuscript.

\section{References}

1. Global Change Data Lab (GCDL). Cumulative confirmed COVID-19 deaths and cases, Feb 16, 2021. Oxford: GCDL. [Accessed: Feb 2020]. Available from: https://ourworldindata. org/grapher/cumulative-deaths-and-cases-covid-19?tab=map \&stackMode $=$ absolute $\&$ time $=$ latest $\&$ country $=\sim B E L \&$ region= World

2. Directorate General Statistics - Statistics Belgium (Statbel). Structure of the population. Brussels: Statbel. Accessed: Feb 2020]. Available from: https://statbel.fgov.be/en/themes/ population/structure-population

3. Cori A, Ferguson NM, Fraser C, Cauchemez S. A new framework and software to estimate time-varying reproduction numbers during epidemics. Am J Epidemiol. 2013;178(9):1505-12. https://doi.org/10.1093/aje/kwt133 PMID: 24043437

4. Zhu Y, Bloxham CJ, Hulme KD, Sinclair JE, Tong ZWM, Steele LE, et al. A meta-analysis on the role of children in SARSCoV-2 in household transmission clusters. Clin Infect Dis. 2020; ciaa1825. https://doi.org/10.1093/cid/ciaa1825 PMID: 33283240

5. Baggio S, L'Huillier AG, Yerly S, Bellon M, Wagner N, Rohr M, et al. Severe acute respiratory syndrome coronavirus 2 (SARSCoV-2) viral load in the upper respiratory tract of children and adults with early acute coronavirus disease 2019 (COVID-19). Clin Infect Dis. 2020; ciaa1157. https://doi.org/10.1093/cid/ ciaa1157 PMID: 32761228 
6. Bi Q, Wu Y, Mei S, Ye C, Zou X, Zhang Z, et al. Epidemiology and transmission of COVID-19 in 391 cases and 1286 of their close contacts in Shenzhen, China: a retrospective cohort study. Lancet Infect Dis. 2020;20(8):911-9. https://doi. org/10.1016/S1473-3099(20)30287-5 PMID: 32353347

7. Zhang J, Litvinova M, Liang Y, Wang Y, Wang W, Zhao S, et al. Changes in contact patterns shape the dynamics of the COVID-19 outbreak in China. Science. 2020;368(6498):1481-6.

8. Viner RM, Mytton OT, Bonell C, Melendez-Torres GJ, Ward J, Hudson L, et al. Susceptibility to SARS-CoV-2 infection among children and adolescents compared with adults. JAMA Pediatr. 2021;175(2):143-56. https://doi.org/10.1001/ jamapediatrics.2020.4573 PMID: 32975552

9. Munday JD, Sherratt K, Meakin S, Endo A, B Pearson CA, Hellewell J, et al. Implications of the school-household network structure on SARS-CoV-2 transmission under different school reopening strategies in England. medRxiv. 2020; 2020.08.21.20167965. doi: https://doi.org/10.1101/2020.08.2 1.20167965

10. Davies NG, Klepac P, Liu Y, Prem K, Jit M, Eggo RM. Agedependent effects in the transmission and control of COVID-19 epidemics. Nat Med. 2020;26:1205-11. https://doi. org/10.1038/s41591-020-0962-9

11. Sun K, Wang W, Gao L, Wang Y, Luo K, Ren L, et al. Transmission heterogeneities, kinetics, and controllability of SARS-CoV-2. Science. 2020;371(6526): eabe2424. https://doi. org/10.1126/science.abe2424

12. Davis S, Trapman P, Leirs H, Begon M, Heesterbeek JAP. The abundance threshold for plague as a critical percolation phenomenon. Nature. 2008;454(7204):634-7. https://doi. org/10.1038/nature07053 PMID: 18668107

13. Coletti P, Wambua J, Gimma A, Willem L, Vercruysse S, Vanhoutte B, et al. CoMix: comparing mixing patterns in the Belgian population during and after lockdown. Sci Rep. 2020;10(1):21885. https://doi.org/10.1038/s41598-020-785407 PMID: 33318521

14. Rai B, Shukla A, Dwivedi LK. Estimates of serial interval for COVID-19: A systematic review and meta-analysis. Clin Epidemiol Glob Health. 2021;9:157-61.

\section{License, supplementary material and copyright}

This is an open-access article distributed under the terms of the Creative Commons Attribution (CC BY 4.0) Licence. You may share and adapt the material, but must give appropriate credit to the source, provide a link to the licence and indicate if changes were made.

Any supplementary material referenced in the article can be found in the online version.

This article is copyright of the authors or their affiliated institutions, 2021. 